\title{
Random Modeling and Control of Nonlinear Active Suspension
}

\author{
Mingyue Cui, Liangchao Geng, and Zhaojing Wu \\ School of Mathematics and Information Science, Yantai University, Yantai, Shandong Province 264005, China \\ Correspondence should be addressed to Mingyue Cui; mingyuecmy@126.com
}

Received 10 March 2017; Accepted 8 May 2017; Published 30 May 2017

Academic Editor: Weihai Zhang

Copyright (C) 2017 Mingyue Cui et al. This is an open access article distributed under the Creative Commons Attribution License, which permits unrestricted use, distribution, and reproduction in any medium, provided the original work is properly cited.

\begin{abstract}
For a quarter car with nonlinear active suspension in rough road, the problem of random modeling and control is considered. According to the relative motion principle, the influence of rough road can be seen as that force is disturbed by the noise and a random model is constructed. By an appropriate transform, the model is transformed into a lower triangular system, which can be used as backstepping method. Then a controller is designed such that the mean square of the state converges to an arbitrarily small neighborhood of zero by tuning design parameters. The simulation results illustrate the effectiveness of the proposed scheme. Therefore, the active suspension system offers better riding comfort and vehicle handing to the passengers.
\end{abstract}

\section{Introduction}

The active suspension is the key technology for vehicles to achieve both ride comfort and control performance. Compared with the passive and semiactive suspension system, the active suspension can supply energy from an external source and generate force to achieve the desired performance. Therefore, the performance of active suspension system is better. In recent decades, the control of active suspension systems has been enthusiastically studied by many researchers. Great efforts have been made in modeling and developing control techniques to obtain the ride comfort. Many control methods, for instance, adaptive control [1], PD control [2], robust control [3], fuzzy logic control [4, 5], intelligent control [6], and sliding mode control $[7,8]$, have been recently proposed. However, all of these studies are based on deterministic systems.

In the actual life, the car often runs on rough roads and the influence of the rough road is not negligible. Therefore, it is expected that the passenger still feels comfortable in rough road. For increasing the passenger's comfort, the vertical acceleration of the vehicle caused by road vibrations must be limited which means that the suspension system must absorb the road vibrations and prevent it from transferring to the vehicle body and passengers. It is very necessary to study the random model and control of nonlinear active suspension system in rough road.
In [9-12], the design methods of the controller with different stochastic mechanical systems were studied. However, the stochastic disturbance was described as white noise in these literatures. Because of absorbers, it is more reasonable to describe the final effect of road irregularities as stationary processes. To overcome the conservation, [13] constructed a theoretical framework on stability of random differential equation systems (RDEs) where stochastic disturbance is stationary processes. For a class of Lagrange systems with colored noise, [14] designed a tracking controller such that the mean square of the tracking error converges to an arbitrarily small neighborhood of zero.

Inspired by these, the model and control problem of nonlinear active suspension in rough road are considered in this paper. The main work consists of the following aspects.

(1) Different from the linear deterministic system in [25], the active suspension with nonlinear damper in rough road is considered in this paper, which increases the difficulty of modeling and design.

(2) The main difficulty for dynamics modeling is how to transform the effect of the road irregularities to the suspension. In this paper, regardless of the rough road, dynamics model of the system is firstly constructed. According to the dynamic-static method and the relative motion, the road irregularities are transformed to the force disturbed by the stationary processes. Then the random dynamic model is established. 
(3) Because the system is an underactuated system which is not the quasi lower triangular structure, one cannot use the design method of vector controller in [9-12]. In this paper, according to special form of the model, the system is transformed into a lower triangular system by a transform. Then using backstepping design method, a state feedback controller is designed such that the state can be made arbitrarily small by tuning design parameters. The simulation results illustrate the effectiveness of the proposed scheme.

This paper is organized as follows. In Section 2, the mathematical preparation is given and the problem is formulated. The random model is constructed in Section 3. In Section 4, the tracking controller design and stability analysis are addressed. A simulation result is given in Section 5. Section 6 concludes the paper.

Notations. The following notations are used throughout the paper: For a vector $x, x^{T}$ denotes its transpose; $|\cdot|$ denotes the usual Euclidean norm of "."; $E$ denotes the mathematical expectation; $\mathbb{R}_{+}$denotes the set of all nonnegative real numbers; $\mathbb{R}^{n}$ denotes the real $n$-dimensional space; $\mathbb{R}^{n \times m}$ denotes the real $n \times m$ matrix space; $C^{i}$ denotes the set of all functions with continuous $i$ th partial derivative; $\mathscr{K}$ denotes the set of all functions: $\mathbb{R}_{+} \rightarrow \mathbb{R}_{+}$, which are continuous, strictly increase, and vanish at zero; $\mathscr{K}_{\infty}$ denotes the set of all functions which are of class $\mathscr{K}$ and unbounded. $\mathscr{K} \mathscr{L}$ denotes the set of all functions $\beta(s, t): \mathbb{R}_{+} \times \mathbb{R}_{+} \rightarrow \mathbb{R}_{+}$, which is of class- $\mathscr{K}$ for each fixed $t$ and decreases to zero as $t \rightarrow \infty$ for each fixed $s$. For simplicity, sometimes the arguments of functions are dropped.

\section{Mathematical Preliminaries and Problem Formulation}

2.1. Mathematical Preliminaries. Consider the following random nonlinear affine system:

$$
\dot{x}=f(x, t)+g(x, t) \xi(t), \quad x\left(t_{0}\right)=x_{0},
$$

where $x \in \mathbb{R}^{n}$ is the state of system, $\xi(t) \in \mathbb{R}^{m}$ is a stochastic process, and the underlying complete probability space is taken to be the quartet $\left(\Omega, \mathscr{F}_{t}, \mathscr{F}_{t}, P\right)$ with a filtration $\mathscr{F}_{t}$ satisfying the usual condition (i.e., it is increasing and right continuous while $\mathscr{F}_{0}$ contains all $P$-null sets). Both functions $f: \mathbb{R}^{n} \times \mathbb{R}_{+} \rightarrow \mathbb{R}^{n}$ and $g: \mathbb{R}^{n} \times \mathbb{R}_{+} \rightarrow \mathbb{R}^{n \times m}$ are locally Lipschitz in $x$ piecewise continuous in $t$; that is, for each $R>0$, there exists a constant $L_{R}>0$ such that

$$
\begin{aligned}
& \left|f\left(x_{1}, t\right)-f\left(x_{2}, t\right)\right|+\left|g\left(x_{1}, t\right)-g\left(x_{2}, t\right)\right| \\
& \quad \leq L_{R}\left|x_{2}-x_{1}\right|
\end{aligned}
$$

for any $t \in \mathbb{R}_{+}$and $x_{1}, x_{2} \in U_{R}=\{x:|x| \leq R\}, x_{1} \neq x_{2}$. Moreover, $f(0, t)$ and $g(0, t)$ are bounded a.s.

In order to obtain the stability, process $\xi(t)$ satisfies the following assumption.
Assumption 1. Process $\xi$ is a $\mathscr{F}_{t}$-adapted process and piecewise continuous, and there exist positive constants $c_{0}, d_{0}$ such that

$$
\sup _{t_{0} \leq s \leq t} E|\xi(s)|^{2} \leq d_{0} e^{c_{0} t}, \quad \forall t \geq t_{0}
$$

The following definition, criterion, and inequality are represented now for the stability analysis.

Definition 2 (see [13]). System (1) with Assumption 1 is said to be noise-to-state stable in probability (NSS-P) if there exist a function $\beta(\cdot, \cdot) \in \mathscr{K} \mathscr{L}$ and a function $\gamma(\cdot) \in \mathscr{K}_{\infty}$ such that, for any $\epsilon>0, x_{0} \in \mathbb{R}^{n}, t \geq t_{0}$,

$$
\begin{aligned}
& P\left\{|x(t)| \leq \beta\left(\left|x_{0}\right|, t-t_{0}\right)+\gamma\left(\sup _{t_{0} \leq s \leq t} E|\xi(s)|^{2}\right)\right\} \\
& \quad \geq 1-\epsilon .
\end{aligned}
$$

Lemma 3 (see [13]). Suppose that for system (1) with conditions (3), there exist parameters $c>0$ and $d>0$ and a function $V \in C^{1}$ such that

$$
\begin{gathered}
\alpha_{1}(|x|) \leq V(x) \leq \alpha_{2}(|x|), \\
\dot{V}(x(t)) \leq-c V(x(t))+d|\xi(t)|^{2},
\end{gathered}
$$

where $\alpha_{1}, \alpha_{2}$ are functions of class $\mathscr{K}_{\infty}$. Then system (1) has a unique solution, and the system is NSS-P.

Definition 4 (see [15]). A stochastic process $x(t)$ is said to be bounded in probability if the random variables $|x(t)|$ are bounded in probability uniformly in $t$; that is,

$$
\lim _{R \rightarrow \infty} \sup _{t>0} P\{|x(t)|>R\}=0 .
$$

Lemma 5 (see [13]). Under Assumption 1, if there exist a positive-definite function $V(x, t) \in C$ and a constant $d_{c}>0$ such that

$$
\begin{array}{r}
\lim _{r \rightarrow \infty} \inf _{|x|>r} V(x, t)=\infty, \\
E V(x, t) \leq d_{c},
\end{array}
$$

then system (1) has a unique solution, and the solution is bounded in probability.

Lemma 6 (see [16]). Consider the continuous functions $k(t)$, $h(t)$, and they are integrable over every finite interval. If a continuous function $y(t)$ satisfies the inequality

$$
\dot{y}(t) \leq k(t) y(t)+h(t), \quad \forall t \geq t_{0},
$$

then

$$
y(t) \leq y\left(t_{0}\right) e^{\int_{t_{0}}^{t} k(s) d s}+\int_{t_{0}}^{t} e^{\int_{s}^{t} k(u) d u} h(s) d s, \quad \forall t \geq t_{0} .
$$




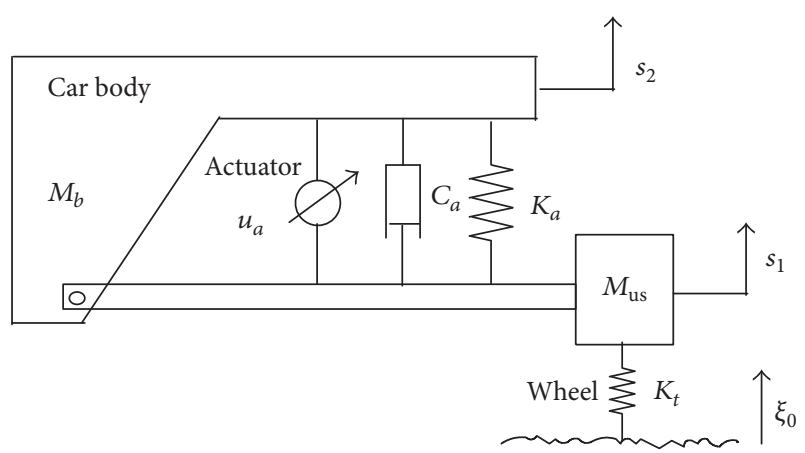

FIGURE 1: The quarter-car model for active suspension design with parallel connection of actuator with spring and damper.

2.2. Problem Formulation. The simplified quarter-car active suspension model is shown in Figure 1 (see [17]). The car runs at a constant speed on a rough road. The road irregularity is described as white noise $\xi_{0}$, which is the acceleration of vertical oscillation. $M_{b}$ is the mass of car body. The wheel is modeled as an unsprung mass $M_{\mathrm{us}}$ with a linear spring $K_{t}$. The actuator is connected in parallel with a linear spring $K_{a}$ and a nonlinear damper $C_{a} \cdot u_{a}$ is the control force from the actuator such as hydraulic actuator. When the components are stationary, the system is in equilibrium. $s_{1}$ and $s_{2}$ denote the barycenter displacements of wheel and vehicle body relative to the equilibrium position, respectively.

The objective of this paper is to design a controller to get more comfortable riding. To this end, two efforts will be taken in the following sections.

(1) Considering the road roughness and the nonlinear damper, construct an appropriate random model to describe the suspension motion of the car.

(2) Design a controller $u_{a}$ such that the states $s_{1}$ and $s_{2}$ can be small as much as possible.

\section{Modeling of Nonlinear Active Suspension System}

Consider the system of particles consisting of the wheel and the car body which are regarded as the mass points, and select $\left(s_{1}, s_{2}\right)$ as the generalized coordinate.

3.1. Modeling under the Assumption That the Road Is Smooth. The total kinetic energy of the system is $K=(1 / 2) M_{\mathrm{us}} \dot{s}_{1}^{2}+$ $(1 / 2) M_{b} \dot{s}_{2}^{2}$. The total potential energy of the system equals $P=(1 / 2) K_{a}\left(s_{2}-s_{1}\right)^{2}+(1 / 2) K_{t} s_{1}^{2}$. Then the Lagrange function

$$
\begin{aligned}
L & =K-P \\
& =\frac{1}{2} M_{b} \dot{s}_{2}^{2}+\frac{1}{2} M_{\mathrm{us}} \dot{s}_{1}^{2}-\frac{1}{2} K_{a}\left(s_{2}-s_{1}\right)^{2}-\frac{1}{2} K_{t} s_{1}^{2} .
\end{aligned}
$$

According to the Lagrangian mechanics [18], the system model is

$$
\begin{array}{r}
M_{\mathrm{us}} \ddot{s}_{1}-K_{a}\left(s_{2}-s_{1}\right)+K_{t} s_{1}=\tau_{1}, \\
M_{b} \ddot{s}_{2}+K_{a}\left(s_{2}-s_{1}\right)=\tau_{2},
\end{array}
$$

where $\tau_{1}$ and $\tau_{2}$ are the generalized forces.
Considering the damper nonlinearity, the Rayleigh dissipation function is

$$
R=\frac{C_{a}}{p+1}\left|\dot{s}_{2}-\dot{s}_{1}\right|^{p+1}
$$

where $p>0$ is an index, which represent the nonlinear form of damping force. Then the dissipative force is

$$
\begin{aligned}
\tau_{d} & =\left(\begin{array}{c}
\tau_{d 1} \\
\tau_{d 2}
\end{array}\right)=-\frac{\partial R}{\partial \dot{s}}=-\left(\begin{array}{c}
\frac{\partial R}{\partial \dot{s}_{1}} \\
\frac{\partial R}{\partial \dot{s}_{2}}
\end{array}\right) \\
& =\left(\begin{array}{c}
C_{a} \operatorname{sign}\left(\dot{s}_{2}-\dot{s}_{1}\right)\left|\dot{s}_{2}-\dot{s}_{1}\right|^{p} \\
-C_{a} \operatorname{sign}\left(\dot{s}_{2}-\dot{s}_{1}\right)\left|\dot{s}_{2}-\dot{s}_{1}\right|^{p}
\end{array}\right),
\end{aligned}
$$

where $\operatorname{sign}(\cdot)$ is the sign function. The control force $u=$ $\left[u_{1}, u_{2}\right]^{T}$, where $u_{1}$ and $u_{2}$ are the control force acting wheel and car body, respectively. Then by replacing $\tau_{i}$ with $\tau_{d i}+u_{i}$, the control system can be modeled as

$$
\begin{aligned}
& M_{\mathrm{us}} \ddot{s}_{1}-K_{a}\left(s_{2}-s_{1}\right)+K_{t} s_{1} \\
& \quad=C_{a} \operatorname{sign}\left(\dot{s}_{2}-\dot{s}_{1}\right)\left|\dot{s}_{2}-\dot{s}_{1}\right|^{p}+u_{1}, \\
& M_{b} \ddot{s}_{2}+K_{a}\left(s_{2}-s_{1}\right) \\
& \quad=-C_{a} \operatorname{sign}\left(\dot{s}_{2}-\dot{s}_{1}\right)\left|\dot{s}_{2}-\dot{s}_{1}\right|^{p}+u_{2} .
\end{aligned}
$$

Remark 7. In this paper, the suspension uses the nonlinear damper. In the dissipative force (13), $p>0$ is an index, which represents the nonlinear form of damping force (see [19]). When $p=1$, it is the common linear damping force.

3.2. Modeling under the Assumption That the Road Is Rough. Road irregularities are often described as white noises. Because of the spring, the final influence of rough road to the wheel and the car body is stationary processes $\xi_{1}$ and $\xi_{2}$, respectively. Therefore, there exists positive constant $K$ such that

$$
\sup _{t_{0} \leq s \leq t} E\left|\xi_{i}(s)\right|^{2} \leq K, \quad \forall t \geq t_{0}, \quad i=1,2
$$

According to dynamic-static method and relative motion [20], the effect of rough road can be seen as the force is disturbed by the noise. Replacing $u_{1}$ and $u_{2}$ with $u_{1}-M_{\mathrm{us}} \xi_{1}$ and $u_{2}-M_{b} \xi_{2}$ in (14) results in

$$
\begin{aligned}
& M_{\mathrm{us}} \ddot{s}_{1}-K_{a}\left(s_{2}-s_{1}\right)+K_{t} s_{1} \\
& \quad=C_{a} \operatorname{sign}\left(\dot{s}_{2}-\dot{s}_{1}\right)\left|\dot{s}_{2}-\dot{s}_{1}\right|^{p}+u_{1}-M_{\mathrm{us}} \xi_{1}, \\
& M_{b} \ddot{s}_{2}+K_{a}\left(s_{2}-s_{1}\right) \\
& \quad=-C_{a} \operatorname{sign}\left(\dot{s}_{2}-\dot{s}_{1}\right)\left|\dot{s}_{2}-\dot{s}_{1}\right|^{p}+u_{2}-M_{b} \xi_{2} .
\end{aligned}
$$


Considering the control force from the actuator, $u_{1}=-u_{a}$ and $u_{2}=u_{a}$. Thus, (16) can be rewritten as

$$
\begin{aligned}
& M_{\mathrm{us}} \ddot{s}_{1}-K_{a}\left(s_{2}-s_{1}\right)+K_{t} s_{1} \\
& \quad=C_{a} \operatorname{sign}\left(\dot{s}_{2}-\dot{s}_{1}\right)\left|\dot{s}_{2}-\dot{s}_{1}\right|^{p}-u_{a}-M_{\mathrm{us}} \xi_{1}, \\
& M_{b} \ddot{s}_{2}+K_{a}\left(s_{2}-s_{1}\right) \\
& \quad=-C_{a} \operatorname{sign}\left(\dot{s}_{2}-\dot{s}_{1}\right)\left|\dot{s}_{2}-\dot{s}_{1}\right|^{p}+u_{a}-M_{b} \xi_{2} .
\end{aligned}
$$

Remark 8. In order to model, the control force $u_{a}$ provided by the actuator can be seen as two independent forces $u_{1}$ and $u_{2}$, which act on the car body and the wheel, respectively. Then according to the Lagrangian mechanics and the dynamicstatic method, the random model (16) is constructed. Finally, considering the constraint $u_{1}=-u_{a}$ and $u_{2}=u_{a}$, the final random model (17) is established.

\section{Control of Nonlinear Active Suspension System}

4.1. Control Design. In order to design controller, choose

$$
\begin{aligned}
& x_{1}=M_{\mathrm{us}} s_{1}+M_{b} s_{2}, \\
& x_{2}=\dot{x}_{1}, \\
& x_{3}=s_{2}-s_{1}, \\
& x_{4}=\dot{x}_{3} .
\end{aligned}
$$

$$
\begin{aligned}
\dot{x}_{1}= & x_{2}, \\
\dot{x}_{2}= & -\frac{K_{t}}{M_{\mathrm{us}}+M_{b}} x_{1}+\frac{K_{t} M_{b}}{M_{\mathrm{us}}+M_{b}} x_{3}-M_{\mathrm{us}} \xi_{1}-M_{b} \xi_{2}, \\
\dot{x}_{3}= & x_{4}, \\
\dot{x}_{4}= & -K_{a}\left(\frac{1}{M_{\mathrm{us}}}+\frac{1}{M_{b}}\right) x_{3}+\frac{K_{t}}{M_{\mathrm{us}}\left(M_{b}+M_{\mathrm{us}}\right)} x_{1} \\
& -\frac{K_{t} M_{b}}{M_{\mathrm{us}}\left(M_{b}+M_{\mathrm{us}}\right)} x_{3} \\
& -C_{a}\left(\frac{1}{M_{\mathrm{us}}}+\frac{1}{M_{b}}\right) \operatorname{sign}\left(x_{4}\right)\left|x_{4}\right|^{p} \\
& +\left(\frac{1}{M_{\mathrm{us}}}+\frac{1}{M_{b}}\right) u_{a}-\xi_{2}+\xi_{1} .
\end{aligned}
$$

Remark 9. Because system (17) is underactuated system which is not transformed into the quasi lower triangular structure, the design methods of vector controller in [912] are not applicable to this system. In order to design the controller with backstepping design method [17], the system is transformed into a lower triangular system by transform (18). step.

Then the backstepping controller can be given step by
Step 1. Introduce the first two error variables

$$
\begin{aligned}
& z_{1}=x_{1}, \\
& z_{2}=x_{2}-\alpha_{1},
\end{aligned}
$$

where $\alpha_{1}$ is a function to be designed. For the Lyapunov function $V_{1}=(1 / 2) z_{1}^{2}$, by choosing $\alpha_{1}=-c_{1} z_{1}$ with design parameter $c_{1}>0$, the derivative of $V_{1}$ is

$$
\dot{V}_{1}=-c_{1} z_{1}^{2}+z_{1} z_{2}
$$

Step 2. Introducing

$$
z_{3}=x_{3}-\alpha_{2}
$$

then

$$
\begin{aligned}
\dot{z}_{2}= & -\frac{K_{t}}{M_{\mathrm{us}}+M_{b}} x_{1}+\frac{K_{t} M_{b}}{M_{\mathrm{us}}+M_{b}}\left(z_{3}+\alpha_{2}\right)+c_{1} x_{2} \\
& -M_{\mathrm{us}} \xi_{1}-M_{b} \xi_{2} .
\end{aligned}
$$

For the Lyapunov function $V_{2}=V_{1}+(1 / 2) z_{2}^{2}$, the derivative of $V_{2}$ is

$$
\begin{aligned}
\dot{V}_{2} & \\
= & -c_{1} z_{1}^{2}+\frac{K_{t} M_{b}}{M_{\mathrm{us}}+M_{b}} z_{2} z_{3} \\
& +z_{2}\left(z_{1}-\frac{K_{t}}{M_{\mathrm{us}}+M_{b}} x_{1}+\frac{K_{t} M_{b}}{M_{\mathrm{us}}+M_{b}} \alpha_{2}+c_{1} x_{2}\right) \\
& -z_{2} M_{\mathrm{us}} \xi_{1}-z_{2} M_{b} \xi_{2} .
\end{aligned}
$$

Applying Young's inequality (for any vectors $x, y \in \mathbb{R}^{n}$ and any scalars $\epsilon>0, p>1$, there holds $x^{T} y \leq\left(\epsilon^{p} / p\right)|x|^{p}+$ $\left(1 / q \epsilon^{q}\right)|y|^{q}$, where $\left.q=p /(p-1)\right)$ to the last two terms in (24), one has

$$
\begin{aligned}
-z_{2} M_{\mathrm{us}} \xi_{1} & \leq d_{1} M_{\mathrm{us}}^{2} z_{2}^{2}+\frac{1}{4 d_{1}} \xi_{1}^{2}, \\
-z_{2} M_{b} \xi_{2} & \leq d_{2} M_{b}^{2} z_{2}^{2}+\frac{1}{4 d_{2}} \xi_{2}^{2},
\end{aligned}
$$

where $d_{1}>0$ and $d_{2}>0$ are design parameters. Substituting (25) into (24), the resulting $\dot{V}_{2}$ is

$$
\begin{aligned}
\dot{V}_{2} & \leq-c_{1} z_{1}^{2}+\frac{K_{t} M_{b}}{M_{\mathrm{us}}+M_{b}} z_{2} z_{3}+z_{2}\left(z_{1}\right. \\
& -\frac{K_{t}}{M_{\mathrm{us}}+M_{b}} x_{1}+\frac{K_{t} M_{b}}{M_{\mathrm{us}}+M_{b}} \alpha_{2}+c_{1} x_{2}+d_{1} M_{\mathrm{us}}^{2} z_{2} \\
& \left.+d_{2} M_{b}^{2} z_{2}\right)+\frac{1}{4 d_{1}} \xi_{1}^{2}+\frac{1}{4 d_{2}} \xi_{2}^{2} .
\end{aligned}
$$

Choose

$$
\begin{aligned}
\alpha_{2} & =\frac{M_{\mathrm{us}}+M_{b}}{K_{t} M_{b}}\left(-z_{1}-c_{2} z_{2}+\frac{K_{t}}{M_{\mathrm{us}}+M_{b}} x_{1}-c_{1} x_{2}\right. \\
& \left.-d_{1} M_{\mathrm{us}}^{2} z_{2}-d_{2} M_{b}^{2} z_{2}\right),
\end{aligned}
$$


where $c_{2}>0$ is a design parameter. Then (26) can be rewritten as

$$
\begin{aligned}
\dot{V}_{2} \leq & -c_{1} z_{1}^{2}-c_{2} z_{2}^{2}+\frac{K_{t} M_{b}}{M_{\mathrm{us}}+M_{b}} z_{2} z_{3}+\frac{1}{4 d_{1}} \xi_{1}^{2} \\
& +\frac{1}{4 d_{2}} \xi_{2}^{2} .
\end{aligned}
$$

Step 3. Introducing

$$
z_{4}=x_{4}-\alpha_{3}
$$

then

$$
\begin{aligned}
\dot{z}_{3} & =z_{4}+\alpha_{3}-\frac{\partial \alpha_{2}}{\partial x_{1}} x_{2}-\frac{\partial \alpha_{2}}{\partial x_{2}}\left(-\frac{K_{t}}{M_{\mathrm{us}}+M_{b}} x_{1}\right. \\
& \left.+\frac{K_{t} M_{b}}{M_{\mathrm{us}}+M_{b}} x_{3}-M_{\mathrm{us}} \xi_{1}-M_{b} \xi_{2}\right) .
\end{aligned}
$$

For the Lyapunov function $V_{3}=V_{2}+(1 / 2) z_{3}^{2}$, the derivative of $V_{3}$ is

$$
\begin{aligned}
\dot{V}_{3} & \leq-c_{1} z_{1}^{2}-c_{2} z_{2}^{2}+z_{3} z_{4}+\frac{1}{4 d_{1}} \xi_{1}^{2}+\frac{1}{4 d_{2}} \xi_{2}^{2} \\
& +z_{3}\left(\frac{K_{t} M_{b}}{M_{\mathrm{us}}+M_{b}} z_{2}+\alpha_{3}-\frac{\partial \alpha_{2}}{\partial x_{1}} x_{2}\right. \\
& \left.-\frac{\partial \alpha_{2}}{\partial x_{2}}\left(-\frac{K_{t}}{M_{\mathrm{us}}+M_{b}} x_{1}+\frac{K_{t} M_{b}}{M_{\mathrm{us}}+M_{b}} x_{3}\right)\right) \\
& -z_{3} \frac{\partial \alpha_{2}}{\partial x_{2}} M_{\mathrm{us}} \xi_{1}-z_{3} \frac{\partial \alpha_{2}}{\partial x_{2}} M_{b} \xi_{2} .
\end{aligned}
$$

Applying Young's inequality to the last two terms in (31), one has

$$
\begin{gathered}
-z_{3} \frac{\partial \alpha_{2}}{\partial x_{2}} M_{\mathrm{us}} \xi_{1} \leq d_{3}\left(\frac{\partial \alpha_{2}}{\partial x_{2}}\right)^{2} M_{\mathrm{us}}^{2} z_{3}^{2}+\frac{1}{4 d_{3}} \xi_{1}^{2}, \\
-z_{3} \frac{\partial \alpha_{2}}{\partial x_{2}} M_{b} \xi_{2} \leq d_{4}\left(\frac{\partial \alpha_{2}}{\partial x_{2}}\right)^{2} M_{b}^{2} z_{3}^{2}+\frac{1}{4 d_{4}} \xi_{2}^{2},
\end{gathered}
$$

where $d_{3}>0$ and $d_{4}>0$ are design parameters. Then (31) can be rewritten as

$$
\begin{aligned}
\dot{V}_{3} & \leq-c_{1} z_{1}^{2}-c_{2} z_{2}^{2}+z_{3} z_{4}+z_{3}\left(\frac{K_{t} M_{b}}{M_{\mathrm{us}}+M_{b}} z_{2}+\alpha_{3}\right. \\
& -\frac{\partial \alpha_{2}}{\partial x_{1}} x_{2}-\frac{\partial \alpha_{2}}{\partial x_{2}}\left(-\frac{K_{t}}{M_{\mathrm{us}}+M_{b}} x_{1}+\frac{K_{t} M_{b}}{M_{\mathrm{us}}+M_{b}} x_{3}\right) \\
& \left.+d_{3}\left(\frac{\partial \alpha_{2}}{\partial x_{2}}\right)^{2} M_{\mathrm{us}}^{2} z_{3}+d_{4}\left(\frac{\partial \alpha_{2}}{\partial x_{2}}\right)^{2} M_{b}^{2} z_{3}\right)+\left(\frac{1}{4 d_{1}}\right. \\
& \left.+\frac{1}{4 d_{3}}\right) \xi_{1}^{2}+\left(\frac{1}{4 d_{2}}+\frac{1}{4 d_{4}}\right) \xi_{2}^{2} .
\end{aligned}
$$

Choose

$$
\begin{aligned}
\alpha_{3}= & -\frac{K_{t} M_{b}}{M_{\mathrm{us}}+M_{b}} z_{2}-c_{3} z_{3}+\frac{\partial \alpha_{2}}{\partial x_{1}} x_{2} \\
& +\frac{\partial \alpha_{2}}{\partial x_{2}}\left(-\frac{K_{t}}{M_{\mathrm{us}}+M_{b}} x_{1}+\frac{K_{t} M_{b}}{M_{\mathrm{us}}+M_{b}} x_{3}\right) \\
& -d_{3}\left(\frac{\partial \alpha_{2}}{\partial x_{2}}\right)^{2} M_{\mathrm{us}}^{2} z_{3}-d_{4}\left(\frac{\partial \alpha_{2}}{\partial x_{2}}\right)^{2} M_{b}^{2} z_{3},
\end{aligned}
$$

where $c_{3}>0$ is a design parameter. Then

$$
\begin{aligned}
\dot{V}_{3} \leq & -c_{1} z_{1}^{2}-c_{2} z_{2}^{2}-c_{3} z_{3}^{2}+z_{3} z_{4}+\left(\frac{1}{4 d_{1}}+\frac{1}{4 d_{3}}\right) \xi_{1}^{2} \\
& +\left(\frac{1}{4 d_{2}}+\frac{1}{4 d_{4}}\right) \xi_{2}^{2} .
\end{aligned}
$$

Step 4. From (29), one has

$$
\begin{aligned}
\dot{z}_{4}= & \psi\left(x_{1}, x_{2}, x_{3}, x_{4}\right)+\left(\frac{1}{M_{\mathrm{us}}}+\frac{1}{M_{b}}\right) u_{a} \\
& +\left(\frac{\partial \alpha_{3}}{\partial x_{2}} M_{\mathrm{us}}+1\right) \xi_{1}+\left(\frac{\partial \alpha_{3}}{\partial x_{2}} M_{b}-1\right) \xi_{2},
\end{aligned}
$$

where $\psi\left(x_{1}, x_{2}, x_{3}, x_{4}\right)=-K_{a}\left(1 / M_{\mathrm{us}}+1 / M_{b}\right) x_{3}+\left(K_{t} /\right.$ $\left.M_{\mathrm{us}}\left(M_{b}+M_{\mathrm{us}}\right)\right) x_{1}-\left(K_{t} M_{b} / M_{\mathrm{us}}\left(M_{b}+M_{\mathrm{us}}\right)\right) x_{3}-C_{a}\left(1 / M_{\mathrm{us}}+\right.$ $\left.1 / M_{b}\right) \operatorname{sign}\left(x_{4}\right)\left|x_{4}\right|^{p}-\left(\partial \alpha_{3} / \partial x_{1}\right) x_{2}-\left(\partial \alpha_{3} / \partial x_{2}\right)\left(-\left(K_{t} /\left(M_{\mathrm{us}}+\right.\right.\right.$ $\left.\left.\left.M_{b}\right)\right) x_{1}+\left(K_{t} M_{b} /\left(M_{\mathrm{us}}+M_{b}\right)\right) x_{3}\right)-\left(\partial \alpha_{3} / \partial x_{3}\right) x_{4}$. For the Lyapunov function $V_{4}=V_{3}+(1 / 2) z_{4}^{2}$, the derivative of $V_{4}$ is

$$
\begin{aligned}
\dot{V}_{4} \leq & -c_{1} z_{1}^{2}-c_{2} z_{2}^{2}-c_{3} z_{3}^{2}+\left(\frac{1}{4 d_{1}}+\frac{1}{4 d_{3}}\right) \xi_{1}^{2} \\
& +\left(\frac{1}{4 d_{2}}+\frac{1}{4 d_{4}}\right) \xi_{2}^{2} \\
& +z_{4}\left(z_{3}+\psi+\left(\frac{1}{M_{\mathrm{us}}}+\frac{1}{M_{b}}\right) u_{a}\right) \\
& +z_{4}\left(\frac{\partial \alpha_{3}}{\partial x_{2}} M_{\mathrm{us}}-1\right) \xi_{1}+z_{4}\left(\frac{\partial \alpha_{3}}{\partial x_{2}} M_{b}-1\right) \xi_{2} .
\end{aligned}
$$

Applying Young's inequality, one has

$$
\begin{aligned}
z_{4}\left(\frac{\partial \alpha_{3}}{\partial x_{2}} M_{\mathrm{us}}+1\right) \xi_{1} \leq & d_{5}\left(\frac{\partial \alpha_{3}}{\partial x_{2}} M_{\mathrm{us}}+1\right)^{2} z_{4}^{2} \\
& +\frac{1}{4 d_{5}} \xi_{1}^{2} \\
z_{4}\left(\frac{\partial \alpha_{3}}{\partial x_{2}} M_{b}-1\right) \xi_{2} \leq & d_{6}\left(\frac{\partial \alpha_{3}}{\partial x_{2}} M_{b}-1\right)^{2} z_{4}^{2} \\
& +\frac{1}{4 d_{6}} \xi_{2}^{2},
\end{aligned}
$$


where $d_{5}>0$ and $d_{6}>0$ are design parameters. Substituting (38) into (37) leads to

$$
\begin{aligned}
\dot{V}_{4} & \leq-c_{1} z_{1}^{2}-c_{2} z_{2}^{2}-c_{3} z_{3}^{2}+z_{4}\left(z_{3}+\psi\right. \\
& +\left(\frac{1}{M_{\mathrm{us}}}+\frac{1}{M_{b}}\right) u_{a}+d_{5}\left(\frac{\partial \alpha_{3}}{\partial x_{2}} M_{\mathrm{us}}+1\right)^{2} z_{4} \\
& \left.+d_{6}\left(\frac{\partial \alpha_{3}}{\partial x_{2}} M_{b}-1\right)^{2} z_{4}\right)+\left(\frac{1}{4 d_{1}}+\frac{1}{4 d_{3}}+\frac{1}{4 d_{5}}\right) \\
& \cdot \xi_{1}^{2}+\left(\frac{1}{4 d_{2}}+\frac{1}{4 d_{4}}+\frac{1}{4 d_{6}}\right) \xi_{2}^{2} .
\end{aligned}
$$

Choose

$$
\begin{aligned}
u_{a} & =\frac{M_{\mathrm{us}} M_{b}}{M_{\mathrm{us}}+M_{b}}\left(-z_{3}-c_{4} z_{4}-\psi\right. \\
& \left.-d_{5}\left(\frac{\partial \alpha_{3}}{\partial x_{2}} M_{\mathrm{us}}+1\right)^{2} z_{4}-d_{6}\left(\frac{\partial \alpha_{3}}{\partial x_{2}} M_{b}-1\right)^{2} z_{4}\right),
\end{aligned}
$$

where $c_{4}>0$ is a design parameter. Then

$$
\begin{aligned}
\dot{V}_{4} \leq & -\sum_{i=1}^{4} c_{i} z_{i}^{2}+\left(\frac{1}{4 d_{1}}+\frac{1}{4 d_{3}}+\frac{1}{4 d_{5}}\right) \xi_{1}^{2} \\
& +\left(\frac{1}{4 d_{2}}+\frac{1}{4 d_{4}}+\frac{1}{4 d_{6}}\right) \xi_{2}^{2} .
\end{aligned}
$$

Up to now, the closed-loop system is obtained

$$
\begin{aligned}
\dot{z}_{1} & =-c_{1} z_{1}+z_{2}, \\
\dot{z}_{2} & =-z_{1}-c_{2} z_{2}+\frac{K_{t} M_{b}}{M_{\mathrm{us}}+M_{b}} z_{3}-\frac{M_{\mathrm{us}}+M_{b}}{K_{t} M_{b}}\left(d_{1} M_{\mathrm{us}}^{2}\right. \\
& \left.+d_{2} M_{b}^{2}\right) z_{2}-M_{\mathrm{us}} \xi_{1}-M_{b} \xi_{2}, \\
\dot{z}_{3} & =-\frac{K_{t} M_{b}}{M_{\mathrm{us}}+M_{b}} z_{2}-c_{3} z_{3}+z_{4}-d_{3}\left(\frac{\partial \alpha_{2}}{\partial x_{2}}\right)^{2} M_{\mathrm{us}}^{2} z_{3} \\
& -d_{4}\left(\frac{\partial \alpha_{2}}{\partial x_{2}}\right)^{2} M_{b}^{2} z_{3}+\frac{\partial \alpha_{2}}{\partial x_{2}}\left(M_{\mathrm{us}} \xi_{1}+M_{b} \xi_{2}\right), \\
\dot{z}_{4} & =-z_{3}-c_{4} z_{4}-\frac{M_{\mathrm{us}} M_{b}}{M_{\mathrm{us}}+M_{b}}\left(d_{5}\left(\frac{\partial \alpha_{3}}{\partial x_{2}} M_{\mathrm{us}}+1\right)^{2}\right. \\
& \left.+d_{6}\left(\frac{\partial \alpha_{3}}{\partial x_{2}} M_{b}-1\right)^{2}\right) z_{4}+\left(\frac{\partial \alpha_{3}}{\partial x_{2}} M_{\mathrm{us}}+1\right) \xi_{1} \\
& +\left(\frac{\partial \alpha_{3}}{\partial x_{2}} M_{b}-1\right) \xi_{2} .
\end{aligned}
$$

\subsection{Stability Analysis}

Theorem 10. Consider the random model (17) of quarter-car active suspension. Under assumption (15), choose controller (40). (i) The closed-loop system (42) is NSS-p and all the signals of the closed-loop system are bounded in probability.

(ii) The state $z=\left[z_{1}, z_{2}, z_{3}, z_{4}\right]^{T}$ of the closed-loop system satisfies

$$
\lim _{t \rightarrow \infty} E|z|^{2} \leq \frac{2 d K}{c}
$$

where the right-hand can be made small enough by tuning parameters.

Proof. Obviously, the functions of the closed-loop system satisfy the local Lipschitz condition. The Lyapunov function for the whole system is

$$
V=V_{4}=\sum_{i=1}^{4} \frac{1}{2} z_{i}^{2}
$$

From (41), one has

$$
\dot{V} \leq-c V+d|\xi|^{2},
$$

where $c=2 \min \left\{c_{1}, c_{2}, c_{3}, c_{4}\right\}, d=\max \left\{1 / 4 d_{1}+1 / 4 d_{3}+\right.$ $\left.1 / 4 d_{5}, 1 / 4 d_{2}+1 / 4 d_{4}+1 / 4 d_{6}\right\}$, and $\xi=\left[\xi_{1}, \xi_{2}\right]^{2}$. From Lemma 3 , the closed-loop system is NSS-P.

Furthermore, by defining $v(t)=E V(z(t))$, from (45), one has

$$
\dot{v}(t)=E \dot{V}(x(t)) \leq-c v(t)+d E|\xi(t)|^{2} .
$$

From Lemma 6, one has

$$
v(t) \leq|v(0)| e^{-c\left(t-t_{0}\right)}+d \sup _{t_{0} \leq s \leq t} E|\xi(s)|^{2},
$$

which together with (15) implies

$$
E V(t) \leq V\left(x_{0}\right) e^{-c\left(t-t_{0}\right)}+\frac{d K}{c} \leq V\left(x_{0}\right)+\frac{d K}{c} .
$$

According to Lemma 5, (48) and the definition of $V, z$ is bounded in probability. By $z_{1}=x_{1}, x_{1}$ is bounded in probability. Since $z_{2}=x_{2}-c_{1} z_{1}, x_{2}$ is bounded in probability, too. Similarly, $x_{3}$ and $x_{4}$ are bounded in probability. Then the control $u$ is also bounded in probability by (40).

From (15), (44), and (47), one has

$$
E|z|^{2} \leq 2 E V(t) \leq 2 V\left(x_{0}\right) e^{-c\left(t-t_{0}\right)}+\frac{2 d K}{c},
$$

which leads to (43). Noting $c=2 \min \left\{c_{1}, c_{2}, c_{3}, c_{4}\right\}, d=$ $\max \left\{1 / 4 d_{1}+1 / 4 d_{3}+1 / 4 d_{5}, 1 / 4 d_{2}+1 / 4 d_{4}+1 / 4 d_{6}\right\}$, it is clear that the right-hand sides of (43) can be made small enough by choosing $c_{i}(i=1, \ldots, 4)$ and $d_{j}(j=1, \ldots, 6)$ large enough.

Remark 11. Since $z_{1}=M_{\mathrm{us}} s_{1}+M_{b} s_{2}, z_{3}=s_{2}-s_{1}-\alpha_{2}=$ $s_{2}-s_{1}-\left(\left(M_{\mathrm{us}}+M_{b}\right) / K_{t} M_{b}\right)\left(-z_{1}-c_{2} z_{2}+\left(K_{t} /\left(M_{\mathrm{us}}+M_{b}\right)\right) z_{1}-\right.$ $\left.c_{1}\left(z_{2}-c_{1} z_{1}\right)-d_{1} M_{\mathrm{us}}^{2} z_{2}-d_{2} M_{b}^{2} z_{2}\right)$, then $s_{1}, s_{2}$ can be small enough by choosing parameters appropriately. As a result, the passenger feels comfortable. From Figure 2, the simulation results also demonstrate the comfortableness. 

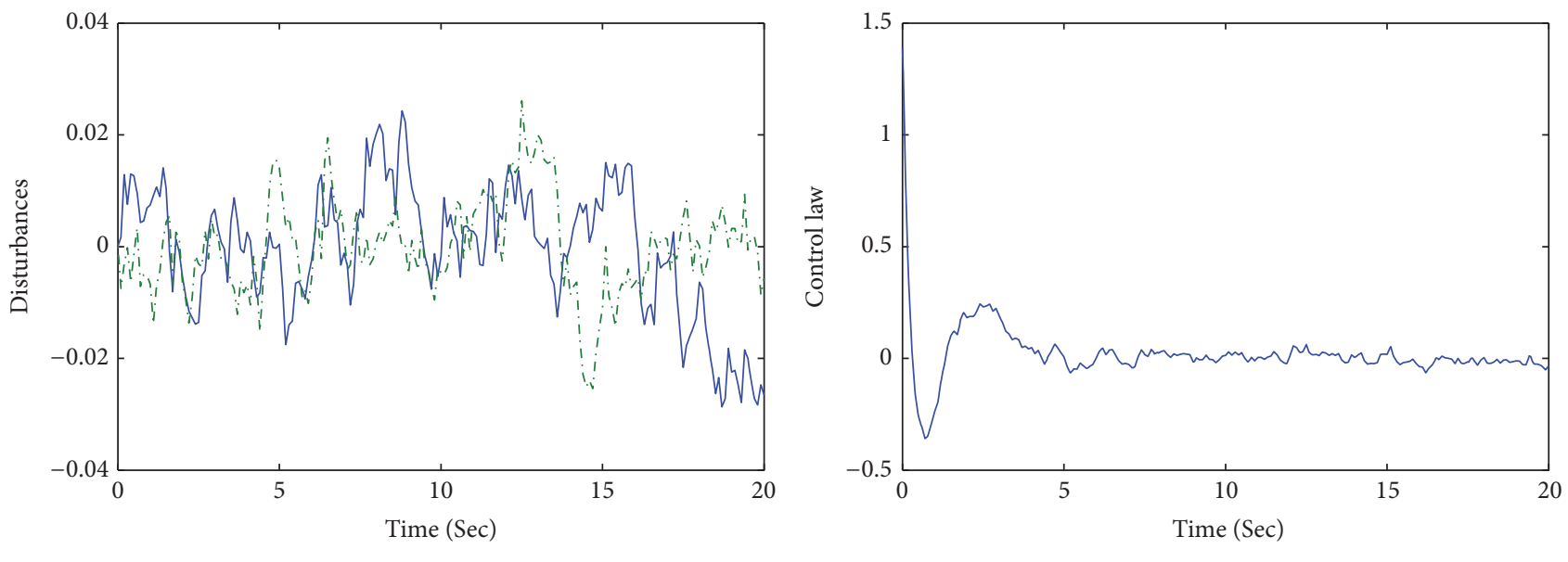

$-\xi_{1}$
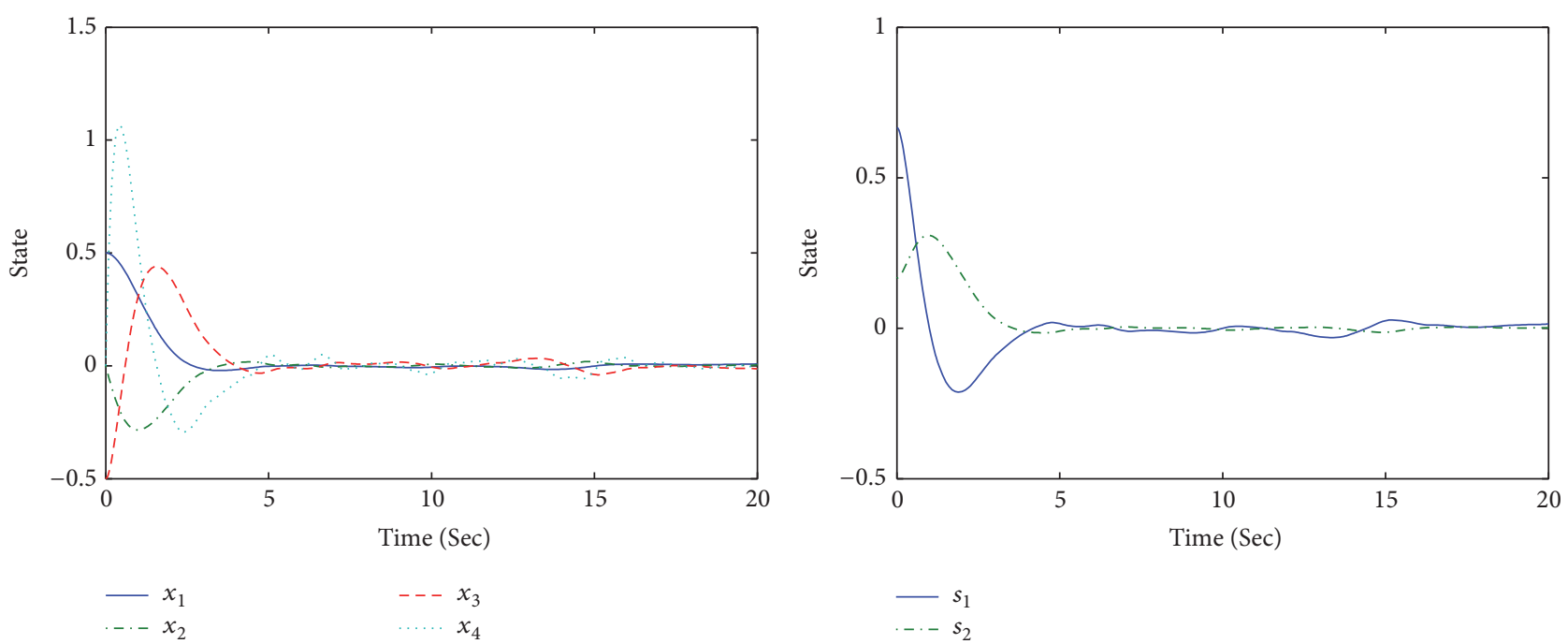

FIGURE 2: The system simulation results.

\section{Simulation Result}

In the simulation, the disturbances $\xi_{1}, \xi_{2}$ are produced by

$$
\dot{\xi}(t)=-\xi(t)+w(t), \quad \xi(0)=0,
$$

where $w(t) \in \mathbb{R}$ is a zero-mean white noise. $\xi=\left(\xi_{1}, \xi_{2}\right)$ is a zero-mean widely stationary process, and $E \xi_{1}^{2}=E \xi_{2}^{2}=0.05$; thus, $K=0.05$. The initial value is $x_{10}=0.5, x_{20}=0, x_{30}=$ -0.5 , and $x_{40}=0$, the parameters of the system are $M_{\mathrm{us}}=0.5$, $M_{b}=1, K_{t}=0.8, K_{a}=0.5, C_{a}=0.1$, and $p=3$, and design parameters are $c_{1}=0.5, c_{2}=1, c_{3}=0.8, c_{4}=1.5, d_{1}=0.2$, $d_{2}=0.1, d_{3}=0.2, d_{4}=0.2, d_{5}=0.2$, and $d_{6}=0.1$.

The simulation result demonstrates the effectiveness of the control scheme.

\section{Conclusion}

The stochastic modeling and control of a quarter car with active suspension in rough road are considered in the paper.
According to the relative motion principle, the influence of rough road is regarded as the force, which is disturbed by the noise. Then a stochastic model is constructed. Based on the model, using backstepping method, a controller is designed such that the mean square of the state converges to an arbitrarily small neighborhood of zero by tuning design parameters. The simulation results illustrate the effectiveness of the proposed scheme.

\section{Conflicts of Interest}

The authors declare that they have no conflicts of interest.

\section{Acknowledgments}

This work is supported by National Natural Science Foundation (NNSF) of China under Grant 61503318 and the Shandong Provincial Natural Science Foundation of China under Grant ZR2014FQ017. 


\section{References}

[1] A. Alleyne and J. K. Hedrick, "Nonlinear adaptive control of active suspensions," IEEE Transactions on Control Systems Technology, vol. 3, no. 1, pp. 94-101, 1995.

[2] J. Lin, K. W. E. Cheng, Z. Zhang, N. C. Cheung, X. Xue, and T. W. Ng, "Active suspension system based on linear switched reluctance actuator and control schemes," IEEE Transactions on Vehicular Technology, vol. 62, no. 2, pp. 562-572, 2013.

[3] T. P. J. Van Der Sande, B. L. J. Gysen, I. J. M. Besselink, J. J. H. Paulides, E. A. Lomonova, and H. Nijmeijer, "Robust control of an electromagnetic active suspension system: Simulations and measurements," Mechatronics, vol. 23, no. 2, pp. 204-212, 2013.

[4] W.-Y. Wang, M.-C. Chen, and S.-F. Su, "Hierarchical T-S fuzzyneural control of anti-lock braking system and active suspension in a vehicle," Automatica. A Journal of IFAC, the International Federation of Automatic Control, vol. 48, no. 8, pp. 16981706, 2012.

[5] H. Li, H. Liu, H. Gao, and P. Shi, "Reliable fuzzy control for active suspension systems with actuator delay and fault," IEEE Transactions on Fuzzy Systems, vol. 20, no. 2, pp. 342-357, 2012.

[6] J. Lin and R.-J. Lian, "Intelligent control of active suspension systems," IEEE Transactions on Industrial Electronics, vol. 58, no. 2, pp. 618-628, 2011.

[7] R.-J. Lian, "Enhanced adaptive self-organizing fuzzy slidingmode controller for active suspension systems," IEEE Transactions on Industrial Electronics, vol. 60, no. 3, pp. 958-968, 2013.

[8] H. Li, J. Yu, C. Hilton, and H. Liu, "Adaptive sliding-mode control for nonlinear active suspension vehicle systems using TS fuzzy approach," IEEE Transactions on Industrial Electronics, vol. 60, no. 8, pp. 3328-3338, 2013.

[9] Z. Wu, M. Cui, and P. Shi, "Backstepping control in vector form for stochastic Hamiltonian systems," SIAM Journal on Control and Optimization, vol. 50, no. 2, pp. 925-942, 2012.

[10] M.-Y. Cui, Z.-J. Wu, X.-J. Xie, and P. Shi, "Modeling and adaptive tracking for a class of stochastic Lagrangian control systems," Automatica. A Journal of IFAC, the International Federation of Automatic Control, vol. 49, no. 3, pp. 770-779, 2013.

[11] M.-Y. Cui, X.-J. Xie, and Z.-J. Wu, "Dynamics modeling and tracking control of robot manipulators in random vibration environment," Institute of Electrical and Electronics Engineers. Transactions on Automatic Control, vol. 58, no. 6, pp. 1540-1545, 2013.

[12] M.-Y. Cui, Z.-J. Wu, and X.-J. Xie, "Output feedback tracking control of stochastic Lagrangian systems and its application," Automatica. A Journal of IFAC, the International Federation of Automatic Control, vol. 50, no. 5, pp. 1424-1433, 2014.

[13] Z. Wu, "Stability criteria of random nonlinear systems and their applications," Institute of Electrical and Electronics Engineers. Transactions on Automatic Control, vol. 60, no. 4, pp. 1038-1049, 2015.

[14] M. Y. Cui, S. T. Wang, and L. C. Geng, "Tracking control for a class of random Lagrange systems with colored noise," in Proceedings of the 35th Chinese Control Conference (CCC), pp. 1814-1819, Chengdu, China, July 2016.

[15] R. Z. Khas'minskii, Stochastic Stability of Differential Equations, S \& N International, Rockville, Md, USA, 1980.

[16] P. Ioannou and J. Sun, Robust Adaptive Control, Prentice-Hall, New Jersey, NJ, USA, 1996.

[17] M. Krstic', I. Kanellakopoulos, and P. Kokotovic', Nonlinear and Adaptive Control Design, Jhon wiley, New York, NY, USA, 1995.
[18] M. G. Calkin, Lagrangian and Hamiltonian Mechanics, World Scientific Publishing Co. Pte. Ltd., Singapore, 1996.

[19] S. Yan, E. H. Dowell, and B. Lin, "Effects of nonlinear damping suspension on nonperiodic motions of a flexible rotor in journal bearings," Nonlinear Dynamics, vol. 78, no. 2, pp. 1435-1450, 2014.

[20] D. L. Goodstein, R. P. Olenick, and T. M. Apostol, The Mechanical Universe: Introduction to Mechanics and Heat, Cambridge University Press, 2008. 


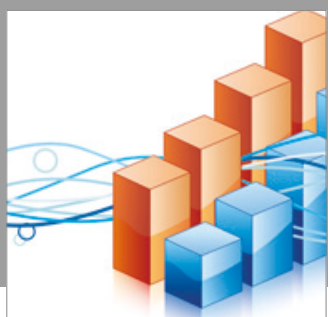

Advances in

Operations Research

vatersals

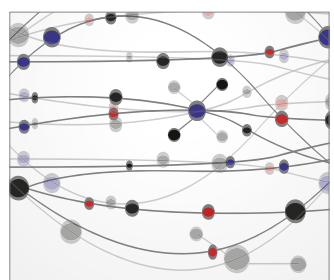

\section{The Scientific} World Journal
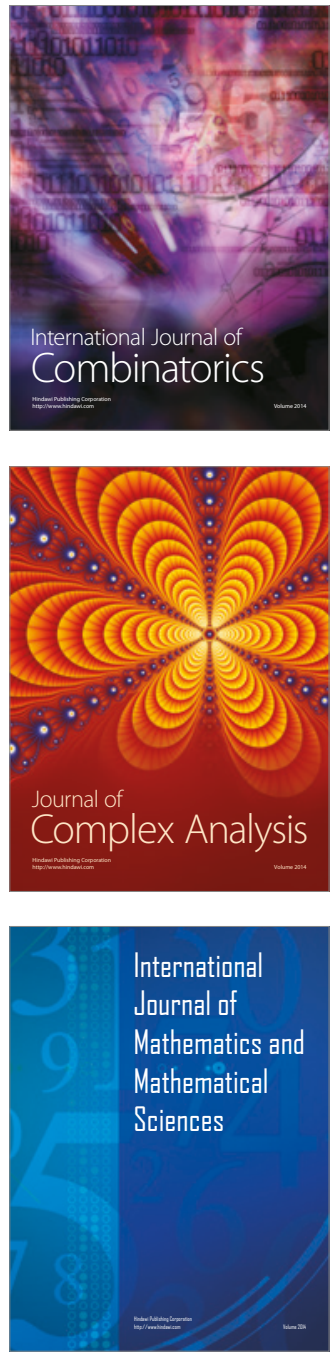
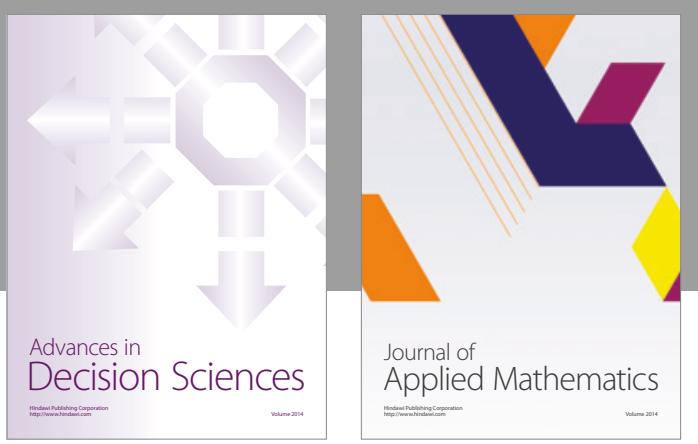

Algebra

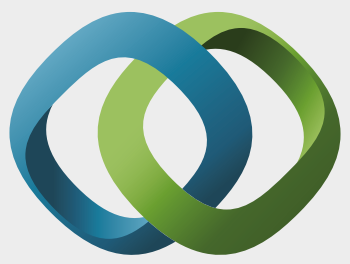

\section{Hindawi}

Submit your manuscripts at

https://www.hindawi.com
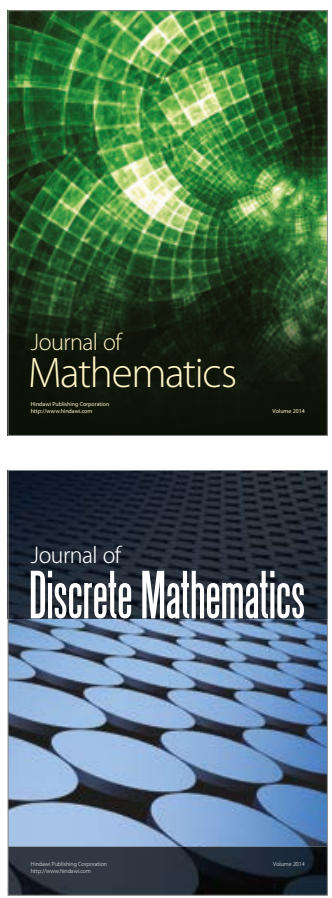

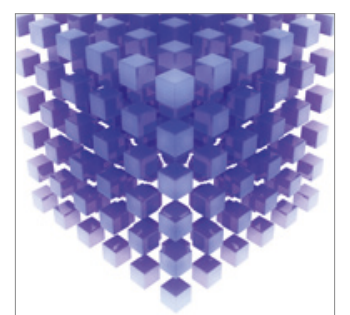

Mathematical Problems in Engineering
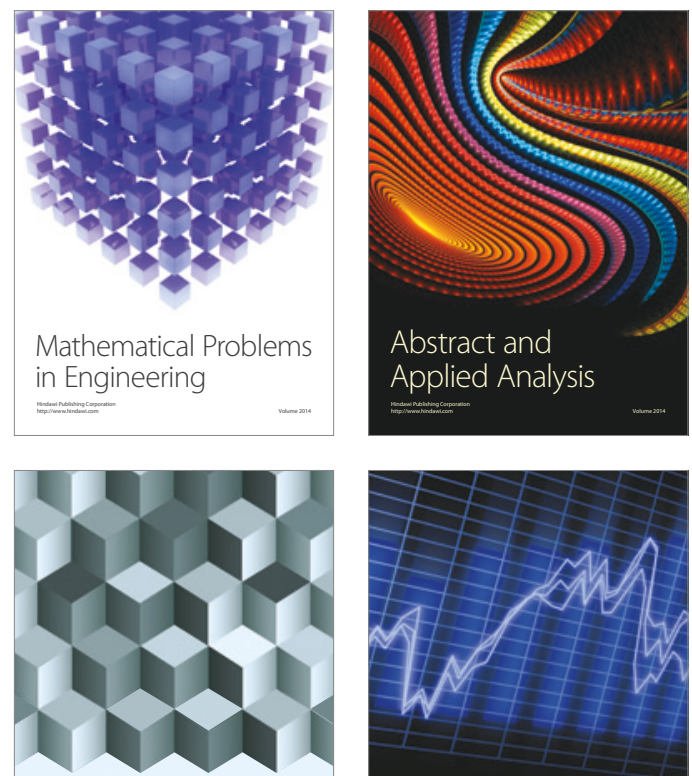

Journal of

Function Spaces

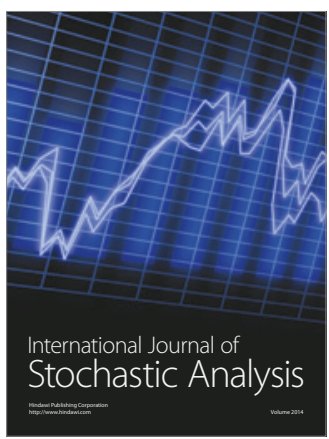

Probability and Statistics
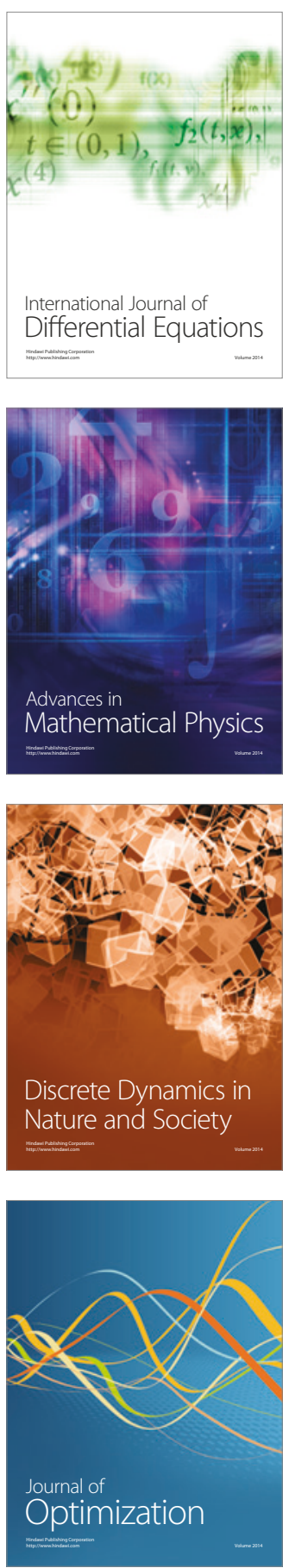